PROCEEDINGS OF THE

AMERICAN MATHEMATICAL SOCIETY

Volume 139, Number 8, August 2011, Pages 2933-2942

S 0002-9939(2010)10714-1

Article electronically published on January 3, 2011

\title{
A CHARACTERIZATION OF THE SINGULAR TIME OF THE MEAN CURVATURE FLOW
}

\author{
ANDREW A. COOPER
}

(Communicated by Richard A. Wentworth)

\begin{abstract}
In this note we investigate the behaviour at finite-time singularities of the mean curvature flow of compact Riemannian submanifolds $M_{t}^{m} \hookrightarrow$ $\left(N^{m+n}, h\right)$. We show that they are characterized by the blow-up of a trace $A=H \cdot$ II of the square of the second fundamental form.
\end{abstract}

\section{INTRODUCTION}

It is well known that the mean curvature flow $\partial_{t} F=H$ of submanifolds $F_{t}: M^{m} \hookrightarrow \mathbb{R}^{m+n}$ has finite-time singularities characterized by the blowup of the second fundamental form II:

Theorem 1.1 (Huisken [4). Suppose $T<\infty$ is the first singular time for a compact mean curvature flow. Then $\max _{M_{t}}|\mathrm{II}| \rightarrow \infty$ as $t \rightarrow T$.

We will prove that in fact it suffices to consider the tensor $A_{i j}=H^{\alpha} h_{i j \alpha}$, where $H=\operatorname{tr}$ II is the mean curvature and $h$ are the components of II:

Theorem 1.2. Let $(N, h)$ be a Riemannian manifold with bounded geometry. Suppose $T<\infty$ is the first singular time for a mean curvature flow of compact submanifolds of $(N, h)$. Then $\max _{M_{t}}|A| \rightarrow \infty$ as $t \rightarrow T$.

By slightly modifying the proof of Theorem 1.2, we also obtain

Theorem 1.3. Suppose that along the flow, $|\mathrm{II}(x, t)|^{p}(T-t) \leq C$ for some $p>1$. Then $\max _{M_{t}}|H| \rightarrow \infty$ as $t \rightarrow T$.

\section{Preliminaries}

First we recall some evolution equations for the flow. We use indices $1 \leq i, j, k, l \leq m, m+1 \leq \alpha, \beta, \gamma \leq m+n$. Let $h_{j \alpha}$ denote the $\alpha$ th component of II $\left(\partial_{i}, \partial_{j}\right)$, let $H^{\alpha}$ denote the $\alpha$ th component of the mean curvature $H$, and let $g_{i j}$ denote the induced metric on $M$. Also, let $\bar{R}$ with four indices denote the Riemannian curvature of $(N, h)$, and let $\bar{R}$ with two indices denote the Ricci curvature of $(N, h)$. Moreover, let $\nabla_{i}$ denote the tangential covariant derivative in the direction

Received by the editors February 24, 2009 and, in revised form, July 31, 2009; February 16, 2010; and July 21, 2010.

2010 Mathematics Subject Classification. Primary 53C44.

Key words and phrases. Mean curvature flow.

The author was partially supported by RTG Research Training in Geometry and Topology NSF grant DMS 0353717 and as a graduate student by NSF grant DMS 06-04759. 
$i$, and let $\bar{\nabla}$ denote the covariant derivative of $h$. We use the summation convention on upper and lower indices.

Lemma 2.1 (Huisken [4], Wang [9]). Along a mean curvature flow $M_{t} \hookrightarrow(N, h)$, we have

(1) $\partial_{t} g_{i j}=-2 H^{\alpha} h_{i j \alpha}$,

(2) $\partial_{t}$ dvol $=-|H|^{2}$ dvol,

$$
\begin{aligned}
\partial_{t} h_{i j \alpha}= & \Delta h_{i j \alpha}+\left(\bar{\nabla}_{k} \bar{R}\right)_{\alpha i j}{ }^{k}+\left(\bar{\nabla}_{j} \bar{R}\right)_{\alpha k i}{ }^{k}-2 \bar{R}_{l i j k} h_{\alpha}{ }^{l k} \\
& +2 \bar{R}_{\alpha \beta j k} h^{k}{ }_{i}{ }^{\beta}+2 \bar{R}_{\alpha \beta i k} h^{k}{ }^{\beta}{ }^{\beta}-\bar{R}_{l k i}{ }^{k} h_{j \alpha}{ }^{l}-\bar{R}_{l k j}{ }^{k} h_{i \alpha}{ }^{l}+\bar{R}_{\alpha k \beta}{ }^{k} h_{i j}{ }^{\beta} \\
& +h_{i l \alpha}\left(h_{l k \gamma} h_{j}{ }^{k \gamma}-h_{k j \gamma} H^{\gamma}\right)+h_{l k \alpha}\left(h^{l k}{ }_{\gamma} h_{i j}{ }^{\gamma}-h_{l j \gamma} h_{i}{ }^{k \gamma}\right) \\
& +h_{i k \beta}\left(h_{l}{ }^{k \beta} h_{\alpha j}{ }^{l}{ }-h_{l j}{ }^{\beta} h_{\alpha}{ }^{l k}\right)-h_{\alpha j k} h_{\beta i}{ }^{k} H^{\beta}+h_{i j \beta}\left\langle e_{\beta}, \bar{\nabla}_{H} e_{\alpha}\right\rangle .
\end{aligned}
$$

By integrating the evolution equation for $\left|\nabla^{s} \mathrm{II}\right|$ and using the Hölder and Morrey inequalities, one can obtain

Theorem 2.2 (Huisken [4]). Along the mean curvature flow, $\sup _{M \times[0, T)}\left|\nabla^{s} \mathrm{II}\right|$ is bounded in terms of $\sup _{M \times[0, T)}|\mathrm{II}|$ and the ambient geometry bounds.

We recall a few lemmas about one-parameter families of Riemannian metrics:

Lemma 2.3 (Glickenstein [2]). Suppose a one-parameter family of complete Riemannian manifolds $(M, g(t))$ is uniformly continuous in $t$; that is, for any $\epsilon>0$ there exists $\delta>0$ so that for any $t_{0},(1-\epsilon) g\left(t_{0}\right) \leq g(t) \leq(1+\epsilon) g\left(t_{0}\right)$ for $t \in\left[t_{0}, t_{0}+\delta\right]$. Then for any $p \in M, r>0$, the metric balls centred at $p$ satisfy

$$
B_{g\left(t_{0}\right)}\left(p, \frac{r}{\sqrt{1+\epsilon}}\right) \subseteq B_{g(t)}(p, r) \subseteq B_{g\left(t_{0}\right)}\left(p, \frac{r}{\sqrt{1-\epsilon}}\right) .
$$

Proof. Let $p, q \in M$. Let $\gamma:[0, S] \rightarrow M$ be a minimising geodesic from $p$ to $q$ for the metric $g\left(t_{0}\right)$. Then the distance $d_{g\left(t_{0}\right)}(p, q)$ in the metric $g\left(t_{0}\right)$ satisfies

$$
\begin{aligned}
d_{g\left(t_{0}\right)}(p, q) & =\int_{0}^{S}|\dot{\gamma}|_{g\left(t_{0}\right)}(s) d s \\
& \geq \frac{1}{\sqrt{1+\epsilon}} \int_{0}^{S}|\dot{\gamma}|_{g(t)}(s) d s \\
& \geq \frac{1}{\sqrt{1+\epsilon}} d_{g(t)}(p, q)
\end{aligned}
$$

so that $\frac{1}{\sqrt{1+\epsilon}} d_{g(t)}(p, q) \leq d_{g\left(t_{0}\right)}(p, q)$. This immediately implies

$$
B_{g\left(t_{0}\right)}\left(p, \frac{r}{\sqrt{1+\epsilon}}\right) \subset B_{g(t)}(p, r) .
$$

The other inclusion is analogous.

Lemma 2.4 (Hamilton [3]). Let $(M, g(t))$ be a one-parameter family of compact Riemannian manifolds defined for $t \in[0, T)$. Suppose that

$$
\int_{0}^{T} \max _{M_{t}}\left|\frac{\partial g}{\partial t}\right|_{g(t)} d t<\infty .
$$


Then the metrics $g(t)$ are uniformly equivalent and converge pointwise as $t \rightarrow T$ to a continuous positive-definite metric $g(T)$.

\section{II AND THE INJECTIVITY RADIUS}

We will prove Theorems 1.2 and 1.3 by a blow-up argument. In particular we will use the Cheeger-Gromov convergence theorem to extract a limit of some submanifolds $F_{j}: M \hookrightarrow \mathbb{R}^{m+n}$, thought of as Riemannian manifolds $\left(M, F_{j}^{*} d x^{2}\right)$. We therefore need the following relationship between injectivity radius and the second fundamental form.

Theorem 3.1. Let $F: M^{m} \rightarrow \mathbb{R}^{m+n}$ be an immersion with $|\mathrm{II}| \leq C$. Then $\operatorname{inj}\left(M, F^{*} d x^{2}\right) \geq \frac{1}{2 \sqrt{2} C}$.

We begin by considering the case of the graph of a map $\psi: \mathbb{R}^{m} \rightarrow \mathbb{R}^{n}$, as in $[7$. We need to compare the standard square-norm of certain objects, e.g. $\left|D^{2} \psi\right|^{2}=$ $\sum_{\substack{1 \leq \alpha \leq n \\ 1 \leq i, j \leq m}}\left(\frac{\partial^{2} \psi_{\alpha}}{\partial x_{i} \partial x_{j}}\right)^{2}$, with the norms of the tensors II and $\nabla$ II in the metric $g$ induced by the immersion. To keep the norms straight, in this section we use $|\cdot|$ for the standard square-norm and $|\cdot|_{g}$ for the norm in $g$ :

$$
\begin{aligned}
|\mathrm{II}|_{g}^{2} & =h_{i j \alpha} h_{k l \beta} g^{\alpha \beta} g^{i k} g^{j l}, \\
|\nabla \mathrm{II}|_{g}^{2} & =\nabla_{i} h_{j k \alpha} \nabla_{p} h_{q r \beta} g^{i p} g^{j q} g^{k r} g^{\alpha \beta} .
\end{aligned}
$$

Lemma 3.2. Let $\psi: D_{r}^{m} \rightarrow \mathbb{R}^{n}$ be a $C^{2}$ function on the disc of radius $r$. Then

$$
\left|D^{2} \psi\right|^{2} \leq\left(1+|D \psi|^{2}\right)^{3}|\mathrm{II}|_{g}^{2}
$$

where II is the second fundamental form of the graph of $\psi$.

Proof. The graph of $\psi$ has immersion map $F\left(x_{1}, \ldots, x_{m}\right)=\left(x_{1}, \ldots, x_{m}, \psi_{1}, \ldots, \psi_{n}\right)$. We use the following tangent and normal frames, where $1 \leq i \leq m$ and $1 \leq \alpha \leq n$ :

$$
\begin{aligned}
e_{i} & =\left(0, \ldots, 0,1,0, \ldots, 0, \frac{\partial \psi_{1}}{\partial x_{i}}, \ldots, \frac{\partial \psi_{n}}{\partial x_{i}}\right)=\left(0, \ldots, 0,1,0, \ldots, 0, D_{i} \psi\right) \\
\nu_{\alpha} & =\left(-\frac{\partial \psi_{\alpha}}{\partial x_{1}}, \ldots,-\frac{\partial \psi_{\alpha}}{\partial x_{m}}, 0, \ldots, 0,1,0, \ldots, 0\right)=\left(-D \psi_{\alpha}, 0, \ldots, 0,1,0, \ldots, 0\right) .
\end{aligned}
$$

These choices induce the metric on the tangent bundle of the graph, which we denote by $g$ with Latin indices:

$$
g_{i j}=e_{i} \cdot e_{j}=\delta_{i j}+D_{i} \psi \cdot D_{j} \psi .
$$

We also get a metric on the normal bundle, which we denote by $g$ with Greek indices:

$$
g_{\alpha \beta}=\nu_{\alpha} \cdot \nu_{\beta}=\delta_{\alpha \beta}+D \psi_{\alpha} \cdot D \psi_{\beta} .
$$

We will use $g^{i j}$ to denote the inverse matrix to $g_{i j}$ and $g^{\alpha \beta}$ to denote the inverse to $g_{\alpha \beta}$. We compute the second fundamental form. Note that $D^{2} F=\left(0, D^{2} \psi\right)$. So we have

$$
\begin{aligned}
\operatorname{II}\left(e_{i}, e_{j}\right) & =\operatorname{proj}^{\perp}\left(D^{2} F\left(e_{i}, e_{j}\right)\right) \\
& =\left(D_{i j}^{2} F \cdot \nu_{\beta}\right) g^{\alpha \beta} \nu_{\alpha} \\
& =\frac{\partial^{2} \psi_{\beta}}{\partial x_{i} \partial x_{j}} g^{\alpha \beta} \nu_{\beta} .
\end{aligned}
$$


In components, $h_{i j \alpha}=\frac{\partial^{2} \psi_{\alpha}}{\partial x_{i} \partial x_{j}}$.

Then the norm-squared of the second fundamental form is

$$
|\mathrm{II}|_{g}^{2}=\frac{\partial^{2} \psi_{\alpha}}{\partial x_{i} \partial x_{j}} \frac{\partial^{2} \psi_{\beta}}{\partial x_{k} \partial x_{l}} g^{\alpha \beta} g^{i k} g^{j l} .
$$

We can think of $|\mathrm{II}|_{g}^{2}$ as the norm-squared of $D^{2} \psi$ in the metric $g$ as opposed to the standard metric. We will compare $g^{\alpha \beta}$ and $g^{i j}$ to the standard metric by giving estimates for the eigenvalues of $g^{\alpha \beta}$ and $g^{i j}$. To do this we estimate the eigenvalues of $g_{i j}$ and $g_{\alpha \beta}$.

Each eigenvalue $\lambda$ of $g_{\alpha \beta}$ has the form $\lambda=g(X, X)=g_{\alpha \beta} X^{\alpha} X^{\beta}$ for some eigenvector $X \in \mathbb{R}^{n}$ with $|X|^{2}=\sum_{\alpha}\left(X^{\alpha}\right)^{2}=1$. Then

$$
\begin{aligned}
\lambda & =\left(\delta_{\alpha \beta}+D \psi_{\alpha} \cdot D \psi_{\beta}\right)\left(X^{\alpha} X^{\beta}\right) \\
& =|X|^{2}+\left(X^{\alpha} D \psi_{\alpha}\right) \cdot\left(X^{\beta} D \psi_{\beta}\right) \\
& =1+\left|X^{\alpha} D \psi_{\alpha}\right|^{2} .
\end{aligned}
$$

Thus $1 \leq \lambda \leq 1+|D \psi|^{2}$. Similarly for an eigenvalue $\mu$ of $g_{i j}$, we have

$$
\begin{aligned}
\mu & =\left(\delta_{i j}+D_{i} \psi \cdot D_{j} \psi\right) X^{i} X^{j} \\
& =|X|^{2}+\left|D_{X} \psi\right|^{2} \\
& =1+\left|D_{X} \psi\right|^{2} .
\end{aligned}
$$

So $1 \leq \mu \leq 1+|D \psi|^{2}$.

Thus the eigenvalues of the inverse matrices $g^{\alpha \beta}$ and $g^{i j}$ are bounded away from zero and infinity:

$$
\begin{aligned}
& 1 \geq\left|\lambda^{-1}\right| \geq \frac{1}{1+|D \psi|^{2}}, \\
& 1 \geq\left|\mu^{-1}\right| \geq \frac{1}{1+|D \psi|^{2}} .
\end{aligned}
$$

So we can estimate

$$
\begin{aligned}
|\mathrm{II}|_{g}^{2} & =\frac{\partial^{2} \psi_{\alpha}}{\partial x_{i} \partial x_{j}} \frac{\partial^{2} \psi_{\beta}}{\partial x_{k} \partial x_{l}} g^{\alpha \beta} g^{i k} g^{j l} \\
& \geq \sum_{\substack{1 \leq \alpha \leq n \\
1 \leq i, j \leq m}}\left(\frac{\partial^{2} \psi_{\alpha}}{\partial x_{i} \partial x_{j}}\right)^{2} \frac{1}{\left(1+|D \psi|^{2}\right)\left(1+|D \psi|^{2}\right)^{2}} \\
& =\left|D^{2} \psi\right|^{2} \frac{1}{\left(1+|D \psi|^{2}\right)^{3}},
\end{aligned}
$$

which establishes our lemma.

Our next step is to show that any immersed submanifold $F: M \leftrightarrow \mathbb{R}^{m+n}$ can be written as a collection of graphs of functions $\psi$ with small $|D \psi|$.

We introduce the following notation and notions, following [5]. Given $q \in M$, denote by $A_{q}$ any Euclidean isometry which takes $F(q)$ to the origin and $T_{F(q)} F(M)$ to the plane $\left\{\left(x_{1}, \ldots, x_{m}, 0\right)\right\}$. Let $\pi$ be the projection of $\mathbb{R}^{n+m}$ to the plane $\left\{\left(x_{1}, \ldots, x_{m}, 0\right)\right\}$. Define $U_{r, q} \subset M$ to be the component of $\left(\pi \circ A_{q}^{-1} \circ F\right)^{-1}\left(D_{r}\right)$ which contains $q$. We call $F: M \leftrightarrow \mathbb{R}^{n+m}$ a $(r, \alpha)$-immersion if for each $q \in M$ there is some $\psi_{q}: D_{r}^{m} \rightarrow \mathbb{R}^{n}$ with $\left|D \psi_{q}\right| \leq \alpha$ so that $A_{q}^{-1} \circ F\left(U_{r, q}\right)=\operatorname{graph}\left(\psi_{q}\right)$. 
Lemma 3.3. Let $0<\alpha \leq 1$. Then for any $C^{2}$-immersed submanifold $F: M^{m} \rightarrow$ $\mathbb{R}^{n+m}$ and any $r$ satisfying

$$
r \leq \frac{\alpha}{\left(1+\alpha^{2}\right)^{3 / 2}} \frac{1}{\sup _{M}|\mathrm{II}|_{g}}
$$

$F$ is an $(r, \alpha)$-immersion.

Proof. Let $q \in M$ be arbitrary. Every submanifold is locally a graph over its tangent plane; thus $A_{q}\left(F\left(U_{r, q}\right)\right)$ can be written as a graph over $D_{r}$ for small enough $r$. So we set $S_{q}=\sup \left\{r \mid F\left(U_{r, q}\right)=\operatorname{graph}\left(\psi_{r, q}\right)\right\}$. For any large $K$, if $F\left(U_{r, q}\right)=\operatorname{graph}\left(\psi_{r, q}\right)$ and $\left|D \psi_{r, q}\right| \leq \frac{K}{2}$, we can extend $\psi_{r, q}$ to have a larger domain and still have $|D \psi| \leq$ $K$. Thus we have

$$
\lim _{r \rightarrow S_{q}} \inf _{\psi} \sup _{D_{r}}|D \psi|=\infty
$$

where the infimum is taken over all $\psi$ of which $F\left(U_{r, q}\right)$ is a graph. Thus for our given $\alpha$ there exist some $r_{q}, \psi_{q}: D_{r_{q}} \rightarrow \mathbb{R}^{n}$ with $\sup _{D_{r_{q}}}\left|D \psi_{q}\right|=\alpha$. Now we use the fundamental theorem of calculus and Lemma 3.2 to get

$$
\alpha=\sup _{D_{r_{q}}}\left|D \psi_{q}\right| \leq r \sup _{D_{r_{q}}}\left|D^{2} \psi_{q}\right| \leq r_{q}\left(1+\alpha^{2}\right)^{3 / 2} \sup _{D_{r_{q}}}\left|\mathrm{II}_{\psi_{q}}\right| g,
$$

which implies that

$$
r_{q} \geq \frac{\alpha}{\left(1+\alpha^{2}\right)^{3 / 2}} \frac{1}{\sup _{D_{r_{q}}}\left|\mathrm{II}_{\psi_{q}}\right|_{g}} \geq \frac{\alpha}{\left(1+\alpha^{2}\right)^{3 / 2}} \frac{1}{\sup _{M}|\mathrm{II}|_{g}} .
$$

So for $r$ less than the right-hand side of (3.13), there is some $\psi: D_{r} \rightarrow \mathbb{R}^{m+n}$ which makes $F\left(U_{r, q}\right)$ a graph and $|D \psi| \leq \alpha$.

Proof of Theorem 3.1. Now choose $\alpha=1$, and let $r$ be given by Lemma 3.3 . It is clear that $B(q, r) \subset U_{r, q}$, since $A_{q} F\left(U_{r, q}\right)$ is a graph over a disc of radius $r$. Thus $B(q, r)$ can be written as a graph over the tangent plane, and in particular $\operatorname{inj}(q) \geq r$.

Since $q$ was arbitrary, we have $\operatorname{inj}(M) \geq r>0$.

\section{The tensor $A$ Blows UP}

We will prove Theorem 1.2 by contradiction. To this end, assume $\max _{M}|A(t)| \leq C$ for all $t \in[0, T)$ and that the flow has a singularity at $T<\infty$.

In particular, we have $|H|^{4}=(\operatorname{tr} A)^{2} \leq n|A|^{2} \leq n C^{2}$. So $|H|$ is also bounded along the flow. We will use $C$ to denote this bound as well.

By Theorem [1.1, we know that as $t \rightarrow T, \max _{M}|\mathrm{II}(t)| \rightarrow \infty$. Let $\left(p_{j}, t_{j}\right)$ be a sequence in $M \times[0, T)$ with $t_{j} \rightarrow T$ and $\max _{t \leq t_{j}}|\mathrm{II}|=\left|\mathrm{II}\left(p_{j}, t_{j}\right)\right|=: Q_{j} \rightarrow \infty$.

Since $\left|\partial_{t} F\right|=|H| \leq C$, we know that $F_{t}(M)$ is contained in the $C T$ tubular neighborhood of $F_{0}(M)$. Thus the $F\left(p_{j}, t_{j}\right)$ accumulate. Passing to a subsequence, we have $F\left(p_{j}, t_{j}\right) \rightarrow p_{0}$. For any $R>0$, we may choose a $j_{0}$ so that $F\left(p_{j}, t_{j}\right)$ lies in the ambient ball of radius $R$ about $p_{0}, B^{N}(R)$ for all $j \geq j_{0}$. In particular, we will take $R$ to be less than the injectivity radius of $(N, h)$.

Consider the flows given by scaling the ambient metric by $Q_{j}$ and time by $Q_{j}^{-2}$ :

$$
F_{j}(p, t)=F\left(p, t_{j}+\frac{t}{Q_{j}^{2}}\right): M \hookrightarrow\left(B^{N}, Q_{j}^{2} h\right) .
$$


Lemma 4.1. Each $F_{j}$ is a mean curvature flow on $M \times\left[-Q_{j}^{2} t_{j}, 0\right]$. The second fundamental form of $F_{j}$ is bounded:

$$
\max _{t \leq 0}\left|\mathrm{II}_{j}\right|=\left|\mathrm{II}_{j}\left(p_{j}, 0\right)\right|=1 .
$$

Proof. Clearly $\partial_{t} F_{j}=Q_{j}^{-2} \partial_{t} F$. We need to show that by scaling the ambient metric, we induce the same scaling in $H$. By definition

$$
\begin{aligned}
H_{Q_{j}^{2} h}\left(F_{j}\right) & =\operatorname{tr}_{Q_{j}^{2} h} \operatorname{II}_{Q_{j}^{2} h}\left(F_{j}\right) \\
& =\left(Q_{j}^{2} h\right)^{p q}\left(\partial_{p} \partial_{q} F_{j}\right)^{\perp_{Q_{j}^{2} h}}
\end{aligned}
$$

where $\left(Q_{j}^{2} h\right)^{p q}$ is the inverse matrix of $\left(Q_{j}^{2} h\right)\left(\partial_{p} F_{j}, \partial_{q} F_{j}\right)$ and $\perp_{Q_{j}^{2} h}$ is the projection onto the normal bundle induced from $Q_{j}^{2} h$.

$Q_{j}^{2} h$ induces the same splitting into tangent and normal bundles as $h$, so we have

$$
\begin{aligned}
H_{Q_{j}^{2} h}\left(F_{j}\right) & =Q_{j}^{-2}\left[h^{p q}\left(\partial_{p} \partial_{q} F_{j}\right)^{\perp_{h}}\right] \\
& =Q_{j}^{-2} \operatorname{tr}_{h} \mathrm{II}_{h}\left(F_{j}\right) \\
& =Q_{j}^{-2} H_{h}\left(F_{j}\right) .
\end{aligned}
$$

So $H$ scales as required.

Similarly scaling the ambient metric by $Q_{j}^{2}$ scales $\mid$ II $\mid$ by $Q_{j}^{-1}$, so we have

$$
\begin{aligned}
\max _{t \leq 0}\left|\mathrm{II}_{j}\right| & =\max _{t \leq t_{j}} Q_{j}^{-1}|\mathrm{II}| \\
& =Q_{j}^{-1}\left|\mathrm{II}\left(p_{j}, t_{j}\right)\right|=1 .
\end{aligned}
$$

It is clear that the $\left(B^{N}, Q_{j}^{2} h, p_{0}\right)$ converge in the Cheeger-Gromov sense to $\left(\mathbb{R}^{m+n}, d x^{2}, 0\right)$, where $d x^{2}$ is the Euclidean metric. In particular, we have a monotone exhausting sequence of open sets $V_{j} \subset \mathbb{R}^{m+n}$ and embeddings $\psi_{j}$ : $\left(V_{j}, 0\right) \rightarrow\left(B^{N}, p_{0}\right)$, such that $\psi_{j}^{*}\left(Q_{j}^{2} h\right) \rightarrow d x^{2}$.

Let $s_{0}=-Q_{1}^{2} t_{1}$. After passing to a smaller spatial region $\tilde{M} \subset M$, we can assume $F_{j}\left(\tilde{M} \times\left[s_{0}, 0\right]\right) \subset \psi_{j}\left(V_{j}\right)$. We restrict our argument to this smaller region and write $M$ without confusion.

Define $\tilde{F}_{j}: M \times\left[s_{0}, 0\right] \rightarrow \mathbb{R}^{m+n}$ by $\tilde{F}_{j}=\psi_{j}^{-1} F_{j}$. Each $\tilde{F}_{j}$ is a mean curvature flow with respect to the metric $\psi_{j}^{*}\left(Q_{j}^{2} h\right)$.

The second fundamental forms $\tilde{\mathrm{II}}_{j}$ of the $\tilde{F}_{j}$ are uniformly bounded, so Theorem 2.2 gives uniform bounds on the covariant derivatives of the $\tilde{\mathrm{II}}_{j}$.

Since $\partial_{t} \tilde{F}_{j}=\tilde{H}_{j}$, we get bounds on the time derivative of $\tilde{F}_{j}$. In fact the evolution of $H$ gives a bound

$$
\begin{aligned}
\left|\partial_{t}^{2} \tilde{F}_{j}\right|=\left|\partial_{t} \tilde{H}_{j}\right| & \leq\left|\Delta \tilde{H}_{j}\right|+C_{1}\left|\nabla \tilde{H}_{j}\right|+C_{2}\left|\tilde{H}_{j}\right|\left|\tilde{\mathrm{I}}_{j}\right|^{2} \\
& \leq\left|\nabla^{2} \tilde{\mathrm{I}}_{j}\right|+C_{1}\left|\nabla \tilde{\mathrm{II}}_{j}\right|+C_{2}\left|\tilde{\mathrm{I}}_{j}\right|^{3} .
\end{aligned}
$$

Similarly, any iterated time derivative $\partial_{t}^{s} \tilde{F}_{j}=\partial_{t}^{s-1}\left(\tilde{H}_{j}\right)$ is controlled in terms of $\left|\nabla^{r} \tilde{\mathrm{II}}_{j}\right|$ for $r \leq 2(s-1)$. The mixed derivatives $\partial_{t}^{r} \nabla^{s} \tilde{\mathrm{II}}_{j}$ are similarly controlled by $\left|\nabla^{l} \tilde{\mathrm{II}}_{j}\right|$ for $l \leq 2 r+s$.

Since $Q_{j}^{2} h \rightarrow d x^{2}$ in $C^{k}$ for any $k$, our bounds on the $\left|\nabla^{s} \tilde{\mathrm{II}}_{j}\right|$ give bounds on $\left|\bar{\nabla}^{s} \overline{\mathrm{II}}_{j}\right|$, where $\bar{\nabla}$ and $\overline{\mathrm{II}}_{j}$ are the connection and second fundamental form of $\tilde{F}_{j}$ with respect to the metric $d x^{2}$. 
Let $t \in\left[s_{0}, 0\right]$. Theorem 3.1 gives $\operatorname{inj}\left(M, \tilde{F}_{j}(t)^{*} d x^{2}\right) \geq \frac{1}{2 \sqrt{2}}$. The Gauss equation guarantees that the Riemannian curvature and its covariant derivatives of $\left(M, \tilde{F}_{j}(t)^{*} d x^{2}, p_{j}\right)$ are bounded uniformly in $j$. Thus by Cheeger-Gromov there is a limit Riemannian manifold $\left(M_{\infty}, g_{\infty}(t), p_{\infty}\right)$.

Adapting the ideas of [8], we consider the growth of balls in $\left(M_{\infty}, g_{\infty}(0)\right)$. We will write $g_{\infty}$ for $g_{\infty}(0)$.

Proposition 4.2. $\left(M, g_{\infty}\right)$ has Euclidean volume growth about $p_{\infty}$.

Proof. Let us use the following conventions for balls and volume forms. $B_{\infty}(\rho)$ will denote the metric ball in $g_{\infty}$ centered at $p_{\infty} ; B_{j}(\rho)$ will denote the metric ball in $F_{t_{j}}^{*}\left(Q_{j}^{2} h\right)$ centred at $p_{j} ; B_{t_{j}}(\rho)$ will denote the metric ball in $F_{t_{j}}^{*} h$ centred at $p_{j}$. Also, $\operatorname{vol}_{\infty}$ will denote the volume form of $g_{\infty} ; \operatorname{vol}_{j}$ will denote the volume form of $F_{t_{j}}^{*}\left(Q_{j}^{2} h\right) ; \operatorname{vol}_{t_{j}}$ will denote the volume form of $F_{t_{j}}^{*} h$. Note that

$$
\begin{aligned}
B_{j}(\rho) & =B_{t_{j}}\left(\frac{\rho}{Q_{j}}\right), \\
\operatorname{vol}_{j} & =Q_{j}^{m} \operatorname{vol}_{t_{j}} .
\end{aligned}
$$

We have, for any $r>0$,

$$
\begin{aligned}
\frac{\operatorname{vol}_{\infty}\left(B_{\infty}(r)\right)}{r^{m}} & =\lim _{j} \frac{\operatorname{vol}_{j}\left(B_{j}(r)\right)}{r^{m}} \\
& =\lim _{j} \frac{\operatorname{vol}_{t_{j}}\left(B_{t_{j}}\left(\frac{r}{Q_{j}}\right)\right)}{\left(\frac{r}{Q_{j}}\right)^{m}} .
\end{aligned}
$$

The evolution of $g$ is

$$
\partial_{t} g_{i j}=-2 H^{\alpha} h_{i j \alpha}=-2 A_{i j},
$$

so we have $\left|\partial_{t} g\right| \leq C$, and in particular $g$ is uniformly continuous in time in the sense of Lemma 2.3 .

Thus we may apply Lemma 2.3 to estimate the metric balls at any time $t_{j}$ by the metric ball at time $t_{j_{0}}$, so long as $t_{j}-t_{j_{0}} \leq \delta$. Since $t_{j} \rightarrow T$, we can pick a $j_{0}$ so that this condition holds for all $j \geq j_{0}$. So we can estimate (4.8) by

$$
\lim _{j} \frac{\operatorname{vol}_{t_{j}}\left(B_{t_{j}}\left(\frac{r}{Q_{j}}\right)\right)}{\left(\frac{r}{Q_{j}}\right)^{m}} \leq \lim _{j} \frac{\operatorname{vol}_{t_{j}}\left(B_{t_{j_{0}}}\left(\frac{r}{\sqrt{1-\epsilon} Q_{j}}\right)\right)}{\left(\frac{r}{Q_{j}}\right)^{m}} .
$$

The evolution of the volume form shows that the flow is pointwise volumereducing. So $\operatorname{vol}_{t_{j}} \leq \operatorname{vol}_{t_{j}}$ for $j \geq j_{0}$. Thus we can estimate (4.10) by

$$
\begin{aligned}
\lim _{j} \frac{\operatorname{vol}_{t_{j}}\left(B_{t_{j_{0}}}\left(\frac{r}{\sqrt{1-\epsilon} Q_{j}}\right)\right)}{\left(\frac{r}{Q_{j}}\right)^{m}} & \leq \lim _{j} \frac{\operatorname{vol}_{t_{j_{0}}}\left(B_{t_{j_{0}}}\left(\frac{r}{\sqrt{1-\epsilon} Q_{j}}\right)\right)}{\left(\frac{r}{Q_{j}}\right)^{m}} \\
& =(1-\epsilon)^{-\frac{m}{2}} \lim _{j} \frac{\operatorname{vol}_{t_{j_{0}}}\left(B_{t_{j_{0}}}\left(\frac{r}{\sqrt{1-\epsilon} Q_{j}}\right)\right)}{\left(\frac{r}{\sqrt{1-\epsilon} Q_{j}}\right)^{m}} .
\end{aligned}
$$

The only dependence of the right-hand side on $j$ is in the $Q_{j}$.

The limit on the right-hand side of (4.11) is the local volume comparison at $p_{j_{0}}$ for the Riemannian manifold $\left(M, F_{t_{j}}^{*} h\right)$. It is well-known that this limit is $\omega_{m}$, the volume of the Euclidean unit $m$-ball. Therefore we have

$$
\frac{\operatorname{vol}_{\infty}\left(B_{\infty}(r)\right)}{r^{m}} \leq(1-\epsilon)^{-\frac{m}{2}} \omega_{m}
$$


Since $\epsilon$ was arbitrary, we have shown $\operatorname{vol}_{\infty}\left(B_{\infty}(r)\right) \leq \omega_{m} r^{m}$.

To show the reverse inequality, we make a similar argument starting from (4.8), this time using the first inclusion of Lemma 2.3. We now seek to estimate $\operatorname{vol}_{t_{j}}$ below by $\operatorname{vol}_{t_{j_{0}}}$. Since we have assumed $|H| \leq C$, the evolution of vol implies that

$$
\operatorname{vol}_{t_{j}} \geq e^{-C^{2}\left(t_{j}-t_{j_{0}}\right)} \operatorname{vol}_{t_{j_{0}}},
$$

and taking $j_{0}$ large enough we may ensure that $e^{-C^{2}\left(t_{j}-t_{j_{0}}\right)} \geq 1-\epsilon$. Then we can estimate (4.8) by

$$
\lim _{j} \frac{\operatorname{vol}_{t_{j}}\left(B_{t_{j}}\left(\frac{r}{Q_{j}}\right)\right)}{\left(\frac{r}{Q_{j}}\right)^{m}} \geq \lim _{j}(1-\epsilon)(1+\epsilon)^{-\frac{m}{2}} \frac{\operatorname{vol}_{t_{j_{0}}}\left(B_{t_{j_{0}}}\left(\frac{r}{\sqrt{1+\epsilon} Q_{j}}\right)\right)}{\left(\frac{r}{\sqrt{1+\epsilon} Q_{j}}\right)^{m}} .
$$

Again we can take the limit in $j$ to get

$$
\frac{\operatorname{vol}_{\infty}\left(B_{\infty}(r)\right)}{r^{m}} \geq(1-\epsilon)(1+\epsilon)^{-\frac{m}{2}} \omega_{m} .
$$

Since $\epsilon$ was arbitrary, we have shown $\operatorname{vol}_{\infty}\left(B_{\infty}(r)\right) \geq \omega_{m} r^{m}$.

Proof of Theorem 1.2. To finish the proof of the theorem, we want to use the volume growth of $\left(M_{\infty}, g_{\infty}\right)$ to obtain a contradiction. The expansion for the volume of balls about $p$ in $r$ is

$$
\operatorname{vol}_{\infty}\left(B_{\infty}(r)\right)=\omega_{m} r^{m}\left(1-\frac{R_{\infty}(p)}{6(m+2)} r^{2}+O\left(r^{3}\right)\right)
$$

where $R_{\infty}\left(p_{\infty}\right)$ is the scalar curvature [1. So Proposition 4.2 immediately implies that $R_{\infty}\left(p_{\infty}\right)=0$.

On the other hand, tracing the Gauss equation twice gives that

$$
\begin{aligned}
R_{\infty}\left(p_{\infty}\right) & =\lim _{j} R_{j}\left(p_{j}\right) \\
& =\lim _{j}\left|H_{j}\left(p_{j}\right)\right|^{2}-\left|\mathrm{II}_{j}\left(p_{j}\right)\right|^{2} \\
& \leq \lim _{j} \frac{C}{Q_{j}^{2}}-1 \\
& =-1 .
\end{aligned}
$$

This is the desired contradiction.

\section{A CONDItion FOR THE BLOW UP OF $H$}

Definition 5.1. A singularity at time $T<\infty$ is of type $I$ if $|\operatorname{II}(x, t)|^{2}(T-t) \leq C<$ $\infty$ for all $x \in M_{t}$ and all $t \in[0, T)$.

This is the slowest possible rate of singularity formation and is attained in the case of a shrinking sphere or cylinder.

We can prove that the mean curvature blows up under a slightly more general condition, namely

Theorem 5.1. Suppose that along the flow, $|\operatorname{II}(x, t)|^{p}(T-t) \leq C$ for some $p>1$. Then $\max _{M_{t}}|H| \rightarrow \infty$ as $t \rightarrow T$. 
Proof. For the purpose of contradiction, suppose $|H| \leq C$ all along the flow.

As in the proof of Theorem 1.2, we consider the parabolic rescales

$$
F_{j}(p, t)=F\left(p, t_{j}+\frac{t}{Q_{j}^{2}}\right): M \hookrightarrow\left(N, Q_{j}^{2} h\right)
$$

where $Q_{j}=\max _{t \leq t_{j}}|\mathrm{II}|$. Each $F_{j}$ is a mean curvature flow, and we have

$$
\left|H_{j}\right| \leq \frac{C}{Q_{j}} .
$$

As in the proof of Theorem 1.2, we want to obtain a limit manifold $\left(M_{\infty}, g_{\infty}\right)$ whose volume growth yields a contradiction.

To proceed to a contradiction as in the proof of Theorem 1.2, we need to establish that the balls of the metrics $g(t)$ can be compared as in the conclusion of Lemma2.3.

Consider the evolution of the metric:

$$
\begin{aligned}
\left|\frac{\partial g}{\partial t}\right|=2|A| & \leq 2|H||\mathrm{II}| \\
& \leq 2 C(T-t)^{-\frac{1}{p}} .
\end{aligned}
$$

The function $(T-t)^{-\frac{1}{p}}$ is integrable, so Lemma 2.4 guarantees that the metrics $g(t)$ converge to a positive definite $g(T)$ as $t \nearrow T$. We thus have for any $\varepsilon_{1}>0$ the existence of a $\delta>0$ such that $t \geq T-\delta$ guarantees $1-\varepsilon_{1} \leq \frac{g(t)}{g(T)} \leq 1+\varepsilon_{1}$. If $t, s \geq T-\delta$, then we have $\frac{1-\varepsilon_{1}}{1+\varepsilon_{1}} \leq \frac{g(t)}{g(s)} \leq \frac{1+\varepsilon_{1}}{1-\varepsilon_{1}}$. Choosing the $\delta$ which corresponds to $\varepsilon_{1}=\frac{\epsilon}{2+\epsilon}$ then guarantees that $(1-\epsilon) g(s) \leq g(t) \leq(1+\epsilon) g(s)$.

We may then apply the proof of Lemma 2.3 to estimate balls in the metrics $g\left(t_{j}\right)$ for $j$ sufficiently large and proceed as in the proof of Theorem 1.2, starting from equation (4.10).

Remark 1. The tensors $H$ and $A$ are either both zero or both nonzero. A bound on $A$ implies that $H$ is bounded. Theorem 1.2 says that $H$ cannot decay "too fast" relative to II. The following question presents itself:

Question 1. Suppose $T<\infty$ is the first singular time for a compact mean curvature flow. Is it generally true that $\max _{M_{t}}|H| \rightarrow \infty$ as $t \rightarrow T$ ?

Remark 2. The blowup of the mean curvature in the type I case has been independently discovered by Le and Šešum [6].

\section{ACKNOWLEDGEMENTS}

The author thanks his adviser, Jon Wolfson, for his help and suggestions. He thanks Xiaodong Wang for suggesting this problem. He also thanks the referee for pointing out mistakes in an earlier version of this paper.

\section{REFERENCES}

[1] Sylvestre Gallot, Dominique Hulin, and Jacques Lafontaine. Riemannian Geometry. SpringerVerlag, 1987. MR909697(88k:53001)

[2] David Glickenstein. Precompactness of solutions to the Ricci flow. Geometry and Topology, 7:487-510, 2003. MR2026540 (2004k:53099)

[3] Richard Hamilton. Three-manifolds with positive Ricci curvature. Journal of Differential Geometry, 17:255-306, 1982. MR664497 (84a:53050)

[4] Gerhard Husiken. Flow by mean curvature of convex hypersurfaces into spheres. Journal of Differential Geometry, 20:237-266, 1984. MR772132 (86j:53097) 
[5] Joel Langer. A compactness theorem for surfaces with $l_{p}$-bounded second fundamental form. Mathematische Annalen, 270:223-234, 1985. MR771980 (86i:53035)

[6] N. Q. Le, N. Šešum. The mean curvature at the first singular time of the mean curvature flow, Ann. I. H. Poincaré-AN, 2010, doi:10.1016/j.anihpc.2010.09.002.

[7] Robert C. Reilly. On the Hessian of a function and the curvatures of its graph. Michigan Math. J., 20:373-383, 1973. MR0334045 (48:12364)

[8] Nataša Šešum. Curvature tensor under the Ricci flow. American Journal of Mathematics, 127:1315-1324, 2005. MR2183526 (2006f:53097)

[9] $\mathrm{Mu}$ Tao Wang. Mean curvature flow of surfaces in Einstein four-manifolds, J. Differential Geom., 57:301-338, 2001. MR 1879229 (2003j:53108)

Department of Mathematics, Michigan State University, East Lansing, Michigan 48824

E-mail address: andrew.a.cooper@gmail.com 